\title{
Waste Processing Facility Location Problem by Stochastic Programming: Models and Solutions
}

\author{
Pavel Popela ${ }^{1}$, Dušan Hrabec ${ }^{2}$, Jakub Kůdela ${ }^{1}$, Radovan Šomplák ${ }^{1}$, Martin \\ Pavlas $^{1}$, Jan Roupec ${ }^{1}$, Jan Novotný ${ }^{1}$ \\ ${ }^{1}$ Brno University of Technology, Faculty of Mechanical Engineering, \\ Technická 2, 61669 Brno, Czech Republic \\ \{popela, roupec, pavlas\}@fme.vutbr.cz, jakub.kudela89@gmail.com, \\ somplak@upei.fme.vutbr.cz, \\ iannovot@gmail.com \\ ${ }^{2}$ Tomas Bata University, Faculty of Applied Informatics \\ Nad Stráněmi 4511, 76005 Zlín, Czech Republic \\ hrabec@fai.utb.cz
}

\begin{abstract}
The paper deals with the so-called waste processing facility location problem (FLP), which asks for establishing a set of operational waste processing units, optimal against the total expected cost. We minimize the waste management (WM) expenditure of the waste producers, which is derived from the related waste processing, transportation, and investment costs. We use a stochastic programming approach in recognition of the inherent uncertainties in this area. Two relevant models are presented and discussed in the paper. Initially, we extend the common transportation network flow model with on-and-off wasteprocessing capacities in selected nodes, representing the facility location. Subsequently, we model the randomly-varying production of waste by a scenario-based two-stage stochastic integer linear program. Finally, we employ selected pricing ideas from revenue management to model the behavior of the waste producers, who we assume to be environmentally friendly. The modeling ideas are illustrated on an example of limited size solved in GAMS. Computations on larger instances were realized with traditional and heuristic algorithms, implemented within MATLAB.
\end{abstract}

Keywords: waste processing, facility location problem, stochastic programming, two decision stages, uncertainty modeling, scenarios, mathematical programming algorithms, heuristics, genetic algorithms, GAMS, MATLAB, pricing related ideas

\section{Introduction}

The growing concern for environment leads to integration of new solutions into traditional WM in practice. About 3 billion tonnes of waste are generated in the European Union countries yearly, see [2]. Moreover, due to the population increase, migration of non EU inhabitants, and economic development in the EU countries, the amount of waste generated is rapidly increasing $[3,6]$. Therefore, 
municipal solid waste producers often face problems of insufficiency in available facility capacities to meet future waste disposal demand [8].

Municipal WM consists of various activities that can be clustered into four processing steps: waste generation, collection, transformation, and disposal [5]. This paper deals with the second stage: collection that also involves waste transportation to waste processing units. Hence, we concern on mathematical modeling and related decision support computations for the optimal WM including facility location planning in this step, see, e.g., [6] for an extensive review of WM modeling, and see also [19] for the facility location in the context of so called waste-to-energy plant planning. So, WM decision making problems belong to the class of optimization problems, which importance recently significantly increases in practice. Therefore, mathematical modeling of particular situations and its computational support can help to decision makers with control of the WM as well as to achieve cost savings [4].

Existing modeling and solution challenges are related to the fact that the studied problems often combine deterministic and stochastic parameters together with nonlinear terms and both continuous and discrete decision variables. Since many parameters in such WM system can be uncertain, straightforward applicability of deterministic mathematical programming methods can be doubtful [8]. Thus, to model the real world requirements in a suitable way, stochastic programming approach has been selected and applied in the model building process.

Among the above mentioned problems, we focus on a so called waste processing FLP that defines the task to choose the set of open and running waste processing units in the best way from the total expected cost point of view. Thus, the facility location decisions must be made when a logistics system is started from scratch i.e. when new products or services are launched or when existing product distributions or services are expanded [4]. Specifically, in this paper, we deal only with the waste producer preferences, and so, we minimize the related processing, transportation, and investment costs.

In this paper, the FLP is considered within the transportation network. In general, network design of transportation problems still belongs to interesting research topics in transportation planning [9,23]. Various approaches have been taken to solve network design problems, see [12] and [15] for a review of the network design problems and see [1] for a detailed review of solution techniques. See also [7] for our previous ideas and further references on a hybrid computational approach to network design problems where we deal mostly with switching on and off edges and arcs of the transportation network.

The next sections of the paper are organized as follows. Section 2 describes the developed FLP within waste transportation network design models. Two considered models are subsequently presented, described, and discussed. Firstly, a common transportation network flow is enriched with the on-off waste processing capacities in the chosen nodes to represent the facility location. Then, the randomly varying waste production is modeled by scenarios and two-stage stochastic integer linear program is obtained. As the second step, we suggest to 
model environmental friendly behavior of waste producers by the ideas inspired by utilization pricing mechanisms in operations research problems. Discussed modeling ideas are explained by an explanatory example in Section 2 Results of computations that were realized for various larger instances with utilization of both traditional and heuristical algorithms by using model and algorithm implementations in GAMS and MATLAB are commented in Section 3. Finally, Section 4 concludes the paper and outlines future research directions and suggests some new computational and modeling ideas for future development.

\section{Models and explanatory examples}

In this section, we develop the cost-minimizing stochastic mixed integer nonlinear program for the above mentioned problem in two steps. The introduced models use the following sets of indices, parameters and decision variables. The sets of indices are as follows:

$I$ : set of transportation network related nodes representing places, $i \in I$,

$J$ : set of transportation network related edges representing routes, $j \in J$,

$S$ : set of included scenarios representing uncertainty, $s \in S$.

In this case, we can identify nodes with waste producers, transition places and waste processing units. In addition, we differ between existing processing units and those units that can be newly established. The edges model routes that may serve for transportation of waste. The structural information describing the network is completed with the following input parameters:

$a_{i, j}$ : network description by node-edge incidence matrix,

$b_{i, s}^{-}$: available amount of produced waste in node $i$ for scenario $s$,

$b_{i}^{+}$: available waste processing capacity in node $i$,

$c_{j}$ : cost per transported unit of waste by edge $j$,

$f_{i}:$ cost per processed unit of waste in node $i$,

$g_{i}^{-}$: cost per unprocessed waste left in node $i$,

$g_{i}^{+}$: cost per unit of unused capacity in node $i$,

$h_{i}:$ cost per switched on processing unit in node $i$,

$p_{s}$ : probability of achieving scenario $s$.

We further assume that waste producers considered in our model coordinate their decision steps and behave as one decision maker. So, among the model elements, the following decision variables are included:

$x_{j, s}$ : waste transported by edge $j$ for scenario $s$, bounded by $x_{U, j}$,

$y_{i, s}$ : amount of waste processed in node $i$ by scenario $s$,

$u_{i, s}^{-}$: amount of untransported waste from node $i$ for scenario $s$,

$u_{i, s}^{+}$: amount of unused processing capacity in node $i$ for scenario $s$,

$v_{i, s}^{-}$: amount of waste transported from node $i$ for scenario $s$ (negative),

$v_{i, s}^{+}$: amount of waste transported to node $i$ for scenario $s$,

$\delta_{i}{ }_{i, s}$ : indicator of switching on-off extra waste processing capacity in $i$.

The first model is a scenario-based two-stage mixed integer linear program that is described as follows: 


$$
\begin{array}{rlrl}
\min \sum_{s \in S} p_{s}\left(\sum_{j \in J} c_{j} x_{j, s}+\sum_{i \in I}\left(f_{i} y_{i, s}+g_{i}^{-} u_{i, s}^{-}+g_{i}^{+} u_{i, s}^{+}\right)\right)+\sum_{i \in I} h_{i} \delta_{i} \\
\text { s.t. } \quad & & \forall i \in I, s \in S, \\
\sum_{j \in J: a(i, j)>0} a_{i, j} x_{j, s} & =v_{i, s}^{+}, & & \forall i \in I, s \in S, \\
\sum_{j \in J: a(i, j)<0} a_{i, j} x_{j, s} & =-v_{i, s}^{-}, & & \forall i \in I, s \in S, \\
y_{i, s}+u_{i, s}^{+} & =b_{i}^{+} \delta_{i}, & & \forall i \in I, j \in J, s \in S, \\
-b_{i, s}^{-}+v_{i, s}^{+} & =v_{i, s}^{-}+y_{i, s}+u_{i, s}^{-}, & \forall i \in I, s \in S, \\
x_{j, s}, y_{i, s}, u_{i, s}^{-}, u_{i, s}^{+}, v_{i, s}^{-}, v_{i, s}^{+} & \geq 0, & & \forall j \in J, s \in S, \\
x_{j, s} & \leq x_{U, j}, & & \forall i \in I .
\end{array}
$$

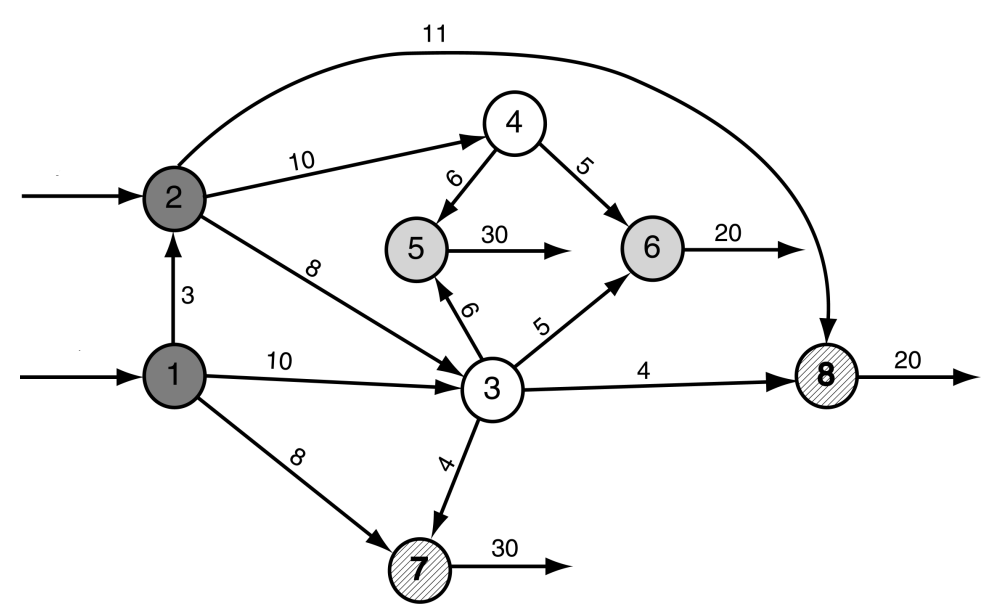

Fig. 1: Test network - visualization of simple input data

The objective function (1) minimizes the total cost that is a sum of scenariorelated costs involving transportation costs, processing costs, penalizing costs for left waste, penalizing costs for unused capacity and investment costs following the investment decisions that must be the same for all scenarios. Eq. (2) means that all flows entering node $i$ are summarized to $v_{i, s}^{+}$. Similarly eq. (3) says that all flows leaving node $i$ are summarized to $v_{i, s}^{-}$. Eq. (4) represents a constraint on the processed amount of waste that is bounded by processing unit capacity. This equation also allows to switch on new waste processing units. To make a difference between already built processing units and newly established ones the value of the first stage decision variables $\delta_{i}$ can be fixed. So, the value 0 is used for transition nodes and value 1 is utilized for the existing processing units. Eq. (5) provides the balance constraint of inputs and outputs in node $i$. Finally, 
(6)-(8) specify domains of the decision variables. For the intial explanation, we have utilized the transportation network on Figure 1. Such a simple example can be solved almost intuitively for one scenario case. Therefore, we list the output for the single scenario in the form of GAMS result file that also contains all input data:

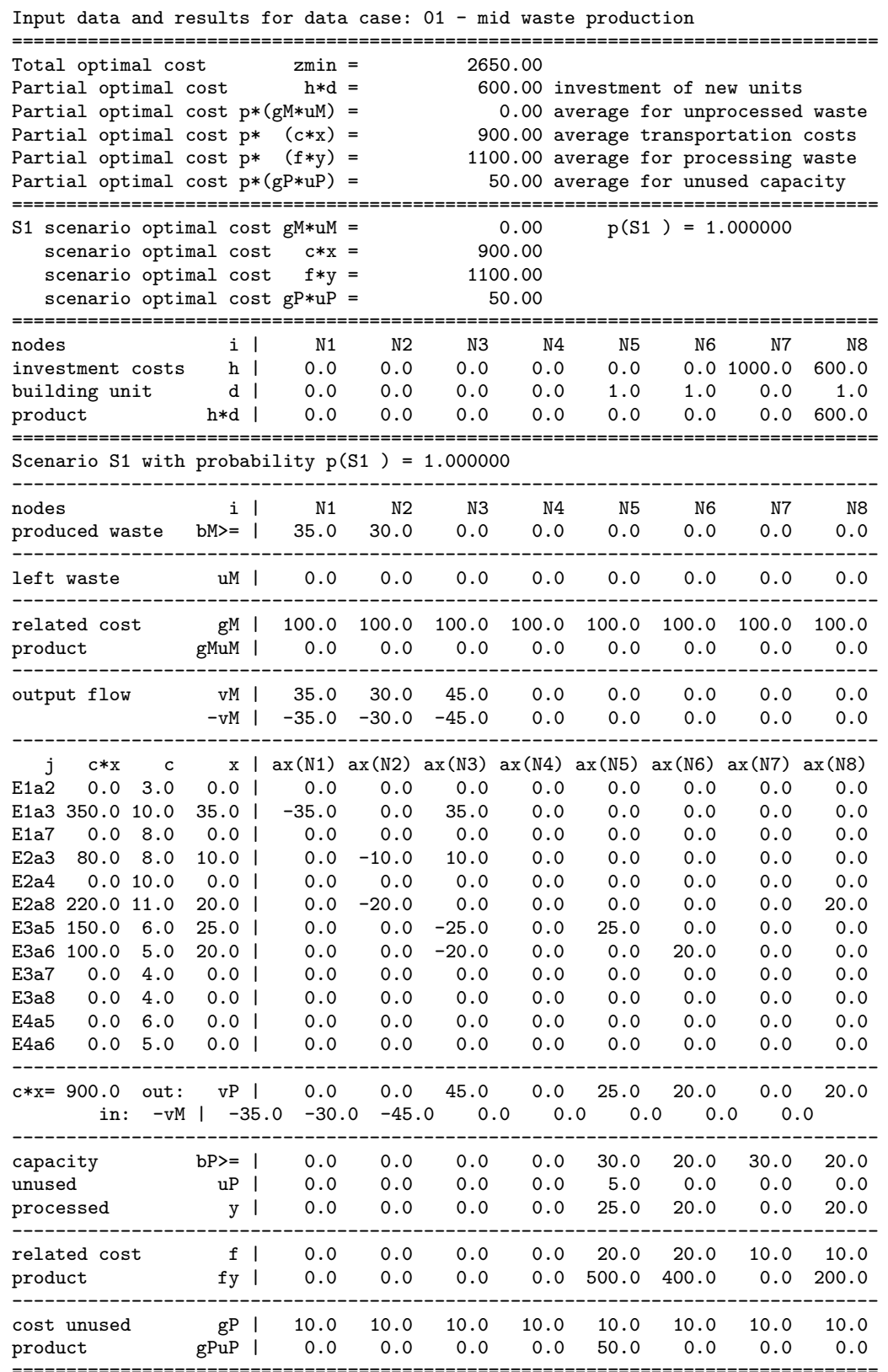




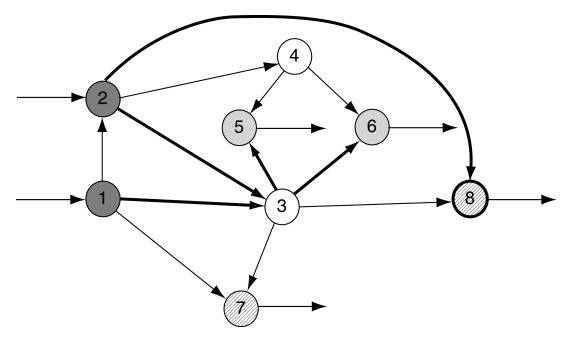

(a) One scenario results

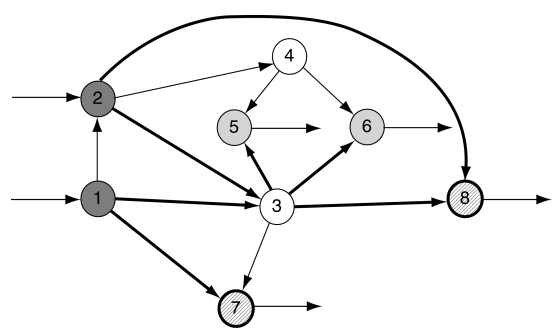

(b) Results for 5 scenarios

Fig. 2: Test network - visualization of results

Additionally, Figure 2a shows the effect of one scenario that leads to the additional switching on available capacity in node 7 (see boldface circle) and extra routes (see boldface edges) used for waste transport. More scenarios taken into the account obviously lead to the increase of newly used processing units (see both nodes 7 and 8) and more routes used for transportation, see Figure $2 \mathrm{~b}$.

To generalize our model, we introduce the pricing related ideas mentioned above in Section 1. Therefore, we assume that waste producers, who are trying to minimize their total cost can improve their behaviour and influence the amount of waste as the decision variable. Consequently, the prices may change. It is reasonable to assume the monopolistic type of behaviour from the set of waste processors and from the government who decide about the related prices. So, the second considered and generalized model is the following scenario-bases twostage stochastic nonlinear mixed integer program:

$$
\begin{aligned}
& \min \sum_{s \in S} p_{s}\left(\sum_{j \in J} c_{j}\left(x_{j, s}\right) x_{j, s}+\right. \\
& \left.\sum_{i \in I}\left(f_{i}\left(y_{i, s}\right) y_{i, s}+g_{i}^{-}\left(\bar{b}_{i}\right) u_{i, s}^{-}+g_{i}^{+} u_{i, s}^{+}\right)\right)+\sum_{i \in I} h_{i} \delta_{i} \\
& \text { s.t. } \quad \sum_{j \in J: a(i, j)>0} a_{i, j} x_{j, s}=v_{i, s}^{+} \text {, } \\
& \sum_{j \in J: a(i, j)<0}^{j \in J: a(i, j)>0} a_{i, j} x_{j, s}=-v_{i, s}^{-}, \quad \forall i \in I, s \in S, \\
& \begin{aligned}
y_{i, s}+u_{i, s}^{+} & =b_{i}^{+} \delta_{i}, \\
-b_{i, s}^{-}+v_{i, s}^{+} & =v_{i, s}^{-}+y_{i, s}+u_{i, s}^{-} \quad \forall i \in I, s \in S, s \in S,
\end{aligned} \\
& \bar{b}_{i}+\varepsilon_{i, s}=b_{i, s}^{-}, \quad \forall i \in I, s \in S, \\
& x_{j, s}, y_{i, s}, u_{i, s}^{-}, u_{i, s}^{+}, v_{i, s}^{-}, v_{i, s}^{+} \geq 0, \quad \forall i \in I, j \in J, s \in S \text {, } \\
& x_{j, s} \leq x_{U, j}, \quad \forall j \in J, s \in S, \\
& \delta_{i} \in\{0,1\}, \quad \forall i \in I, \\
& b_{L, i} \leq \bar{b}_{i} \leq b_{U, i}, b_{i, s}^{-} \geq 0 \text {, }
\end{aligned}
$$

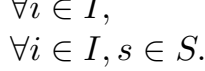


In the second model (9)-(18), most of the constraints (see (10)-(13),(15)(17) and compare it with (2)-(8) ) remain the same, however, several important modifications have been included. The cost coefficients newly depend on decision variables (see the objective function $(9))$ and we have introduced functions $c_{j}\left(x_{j, s}\right), f_{i}\left(y_{i, s}\right)$, and $g_{i}^{-}\left(\bar{b}_{i}\right)$ instead of coefficients $c_{j}, f_{i}$, and $g_{i}^{-}$respectively. We also assume that the decrease of the amount transported or processed will lead to the increase of the related unit cost specified by the price coordinating processing units. Similarly, we assume that the unit governmental penalty for the unprocessed waste will increase with decreasing production of the waste. See Figure 3 for an example of $f_{i}\left(y_{i, s}\right)$ function.

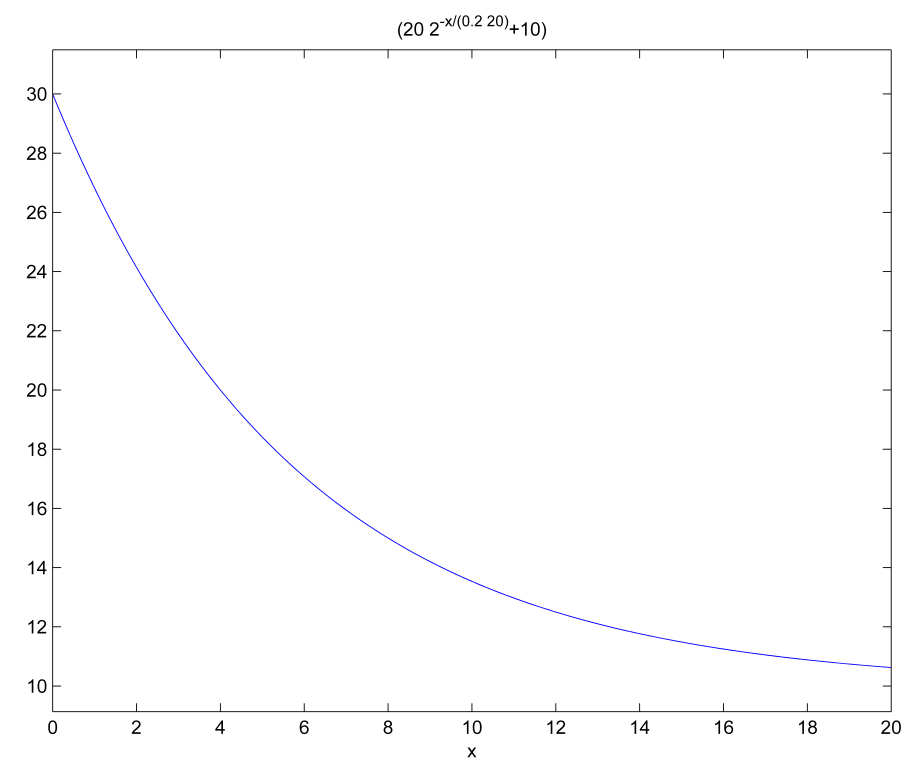

Fig. 3: Modeling pricing ideas

We suggest to notice that under the assumption of strict monotonicity of these functions, so traditional pricing related formulas can appear in the case that we decide to deal with inverse functions. However, the related interpretation derived from the viewpoint of the producers seems to be unrealistic for such case. Therefore, we have converted our original pricing ideas in the final ones that are included in the model. The decision of the waste producers about the amount of the waste delivered for the processing is denoted by $\bar{b}_{i}$ and changes only within the bounds $b_{L, i}$ and $\leq b_{U, i}$ are allowed, see (18). We expect random disturbances following this decision modeled by $\varepsilon_{i, s}$. Then, the $b_{i, s}^{-}$is a dependent variable defined by (14). 


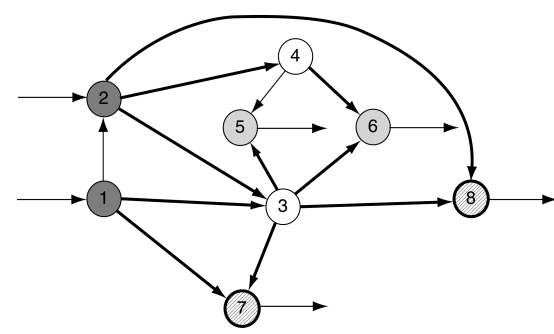

(a) Results for 50 scenarios

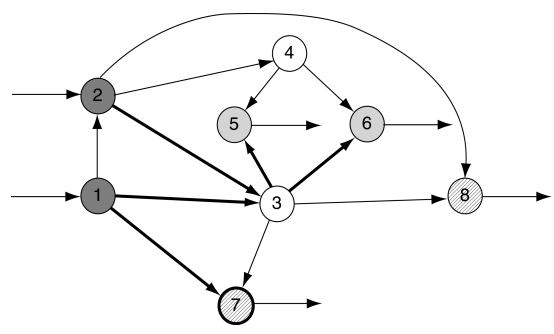

(b) Results for pricing-like mechanism

Fig. 4: Test network - visualization of results

The last figures in this section illustrate the effect of pricing ideas included, see model (9)-(18). Allowing price changes will motivate waste producers to increase, e.g., recycling attempts and it may also reduce their total costs, waste produced and waste processed. Specifically, lessprocessing units must be opened and less routes are used cf. Figures 4a involving solution for 50 scenarios for the first model (1)-(8) and 4b descirbing the results for the second model (9)-(18).

\section{Computations and results}

We have programmed the abovementioned two models in GAMS and we have solved them by the use of BARON, MINOS and CPLEX solvers for small test instances obtaining acceptable results. The next computations were realized for larger instances of the model (9)-(18). However, the solution difficulties have appeared when the original GAMS code was applied as computations have led to increasing computational time needs. Therefore, heuristics have been discussed and the previous authors' ideas related to the suitable hybrid algorithm have been detailed, see [7]. Instead of previous implementations based on combination of the GAMS and $\mathrm{C}++$ codes we have preferred the complete implementation in MATLAB. This implementation combines fmincon function with the genetic algorithm implementation to follow the algorithmic scheme:

1. Set up the instance of scenario-based two-stage mixed integer nonlinear program in MATLAB. Set up control parameters for the genetic algorithm implemented in MATLAB.

2. Create an initial population for GA instance. So, the initial values of $0-$ 1 variables are generated and fixed to obtain a scenario-based (separable) nonlinear program.

3. Several runs of random generators are needed for a specified population size and number of considered scenarios. Repeatedly run fmincon procedure in MATLAB to obtain the set of scenario-related solutions. Each run solves the program for the fixed values of $0-1$ variables.

4. The objective function values are computed, also for new individuals created by means of the genetic operators, initially in 2 . and then in 3 . Store the 
best results obtained from MATLAB (the optimal objective function values and optimal values of all variables for all scenarios) for comparisons.

5. Test the algorithm termination rules and stop in case of their satisfaction. Otherwise continue till the moment when the last scenario solution is obtained.

6. Generate input values for the GA from fmincon results, see step 4. Specifically, the objective function values for each member of population of the GA are obtained from results of the runs in 3.

7. Run GA to update the set of $0-1$ variables (population), see, e.g., [13] for details. Return to step 3.

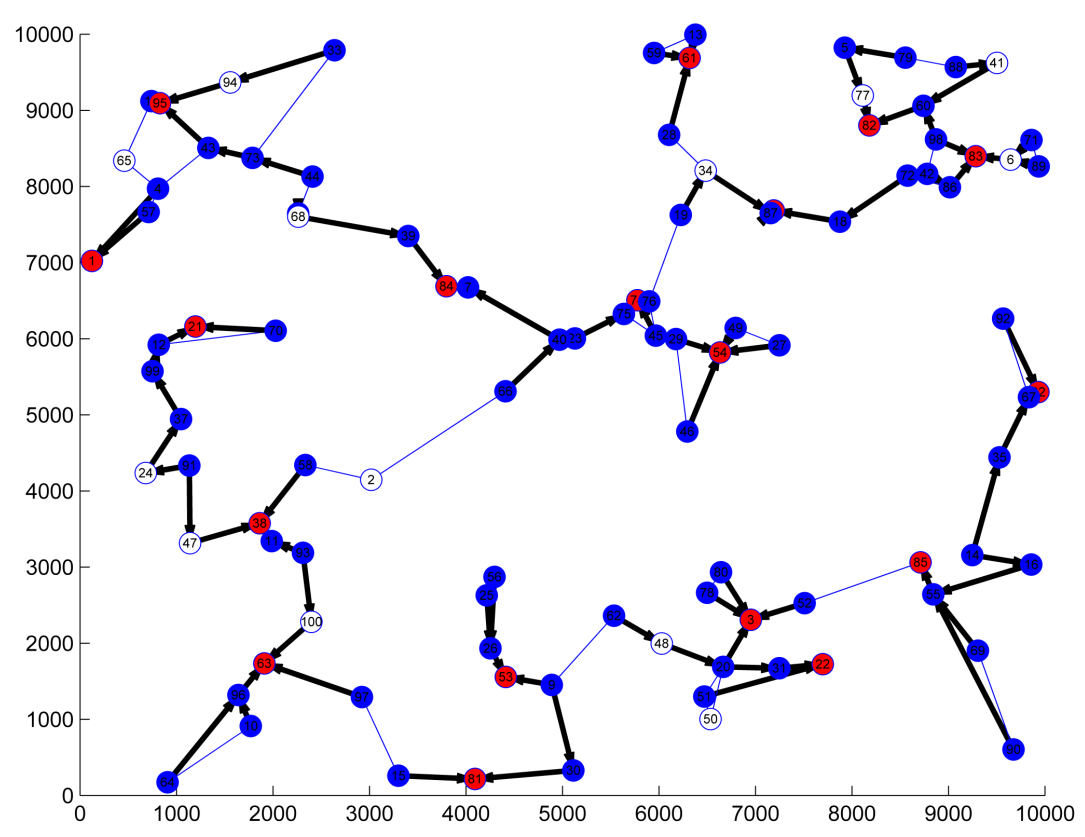

Fig. 5: Visualization of hybrid algorithm results

The results obtained by the hybrid algorithm implementation in MATLAB are illustrated for one instance of data and model (9)-(18) on Figure5. A special postprocessing procedure dynamically supporting the visualization of the obtained results have been implemented in MATLAB as well. Red nodes represent built waste proceessing units (e.g., incinerators), then the blue nodes identify waste producers and white nodes are transition nodes. Let us empahsize that for these test computations to simplify the data instance coding we do not assume any already built waste processing units. Then, the edges are different by the flow. The black edge denotes a non-zero flow while the blue edge identifies a zero 
flow. Similarly, the instances of the various size have been tested and the collected experience is contained in the Table 1 . The average computational times show the expected increasing trends with the increase of number of nodes and number of scenarios.

Table 1: Test results

\begin{tabular}{|c|c|c|c|c|c|c|c|c|}
\hline Number of nodes & \multicolumn{3}{|c|}{10} & \multicolumn{2}{|r|}{20} & \multicolumn{2}{|c|}{40} & 50 \\
\hline Number of scenarios & 1 & 5 & 10 & 1 & 5 & 1 & 3 & 1 \\
\hline Computational time $[\mathrm{s}]$ & 27 & 137 & 184 & 46 & 1070 & 288 & 3027 & 427 \\
\hline Number of nodes & & 12 & & & 24 & & 42 & 55 \\
\hline Number of scenarios & 1 & 5 & 10 & 1 & 5 & 1 & 3 & 1 \\
\hline Computational time [s] & 32 & 151 & 193 & 62 & 1122 & 309 & 3227 & 493 \\
\hline Number of nodes & & 14 & & & 28 & & 44 & 60 \\
\hline Number of scenarios & 1 & 5 & 10 & 1 & 5 & 1 & 3 & 1 \\
\hline Computational time $[\mathrm{s}]$ & 39 & 163 & 205 & 71 & 1197 & 327 & 3302 & 564 \\
\hline Number of nodes & & 16 & & & 32 & & 46 & 65 \\
\hline Number of scenarios & 1 & 5 & 10 & 1 & 5 & 1 & 3 & 1 \\
\hline Computational time $[\mathrm{s}]$ & 45 & 182 & 226 & 83 & 1251 & 339 & 3411 & 617 \\
\hline Number of nodes & & 18 & & & 28 & & 48 & 70 \\
\hline Number of scenarios & 1 & 5 & 10 & 1 & 5 & 1 & 3 & 1 \\
\hline Computational time $[\mathrm{s}]$ & 53 & 199 & 245 & 96 & 1307 & 378 & 3571 & 691 \\
\hline
\end{tabular}

\section{Conclusions and further research}

In the presented paper we have generalized a well known facility location problem to the specific problems of waste processing, see [22] and [19]. We have adopted the standpoint of the waste producers and we minimize the waste-management cost which they face, and which is derived from the related processing, transportation, and investment costs. We have built two stochastic programs starting from the transportation network flow model with on-and-off waste-processing capacities in selected nodes and randomly-varying waste production modelled by scenarios. Then, the pricing ideas from revenue management have been utilized to allow environmentally friendly behaviour of waste producers. For computational purposes a modified hybrid algorithm is implemented in MATLAB and obtained results are visualized.

Further research will lead to adoptation of the models and the algorithm to the case of real-world data and to the viewpoint of the waste processors, see Figure 6. In general, similar mixed integer (either linear, bilinear or nonlinear) stochastic programs may appear in many application areas including design 
problems [20] and [10], control problems [21] or vehicle routing problems [16]. Moreover, a use of more advanced evolutionary algorithms seems to be necessary in further research. Therefore, we refer to genetic [13], differential evolution [17], particle swarm [14] and ant colony [18] optimization algorithms.

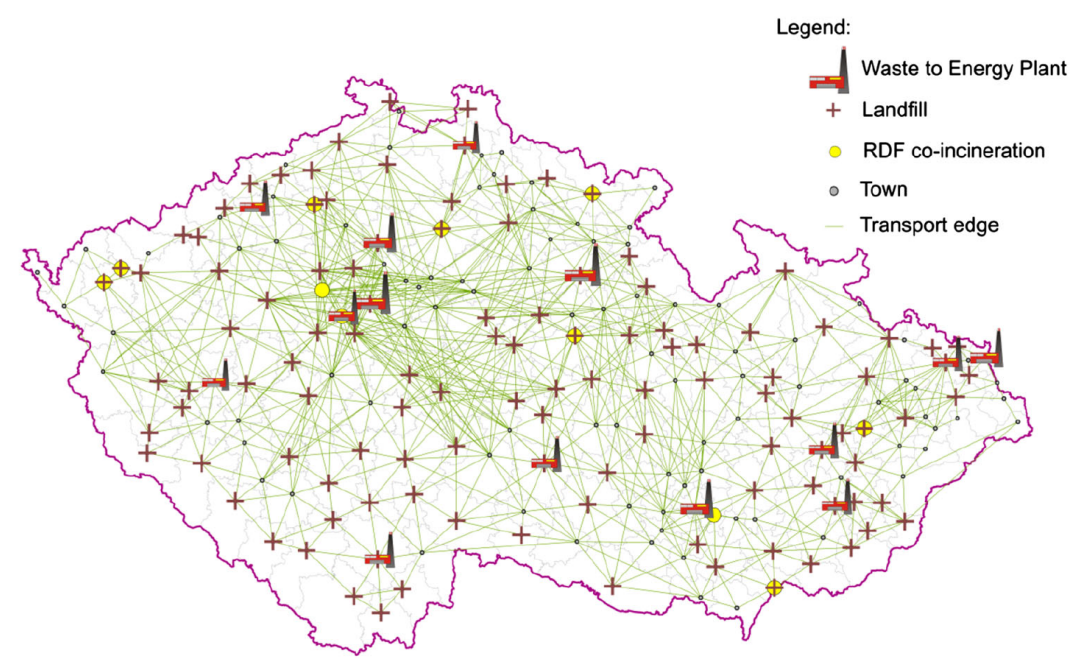

Fig. 6: Real-world transportation network for the Czech Republic

Acknowledgments. This work was supported by the Programme EEA and Norway Grants for funding via grant on Institutional cooperation project nr. NF-CZ07-ICP-4-345-2016 and by the specific research project "Modern Methods of Applied Mathematics for the Use in Technical Sciences", no. FSI-S-142290, id. code 25053. The authors gratefully acknowledge further support from the NETME CENTRE PLUS under the National Sustainability Programme I (Project LO1202) and support provided by Technology Agency of the Czech Republic within the research project No. TE02000236 "Waste-to-Energy (WtE) Competence Centre.

\section{References}

1. Babazadeh, A., Poorzahedy, H., and Nikoosokhan, S.: Application of Particle Swarm Optimization to Transportation Network Design Problem. Journal of King Saud University - Science 23, 293-300 (2011)

2. Blumenthal, K.: Generation and Treatment of Municipal Waste. Technical Report KS-SF-11-031 (2011)

3. Eiselt, H.A., and Marianov, V.: Location modeling for municipal solid waste facilities. Computers \& Operations Research 62, 305-315 (2015)

4. Ghiani, G., Laporte, G., and Musmanno, R.: Introduction to Logistic Systems Planning and Control, Wiley-interscience series in systems and optimization, John Wiley \& Sons, Chichester (2004) 
5. Ghiani, G. et al.: Capacitated location of collection sites in an urban waste management system. Waste Management 32, 1291-1296 (2012)

6. Ghiani, G., Lagana, D., Manni, E., Musmanno, R., and Vigo, D.: Operations research in solid waste management: a survey of strategic and tactical issues. Computers \& Operations Research 44, 22-32 (2014)

7. Hrabec, D., Popela, P., Roupec, J. et al.: Hybrid algorithm for wait-and-see network design problem. 20th International Conference on Soft Computing MENDEL 2014, 97-104. Brno University of Technology, VUT Press, Brno (2014)

8. Huang, G.H., Baetz, B.W., Patry, G.G., and Terluk, V.: Capacity planning for an integrated waste management system under uncertainty: A North American case study. Waste Management \& Research 15, 523-546 (1997)

9. Kaya, O., and Urek, B.: A mixed integer nonlinear programming model and heuristic solutions for location, inventory and pricing decisions in a closed loop supply chain. Computers \& Operations Research 65, 93-103 (2016)

10. Lániková, I. et al.: Optimized design of concrete structures considering environmental aspects. Advances in Structural Engineering 17(4), 495-511 (2014)

11. LeBlanc, L.J.: An Algorithm for Discrete Network Design Problem. Transportation Science 9, 183-199 (1975)

12. Magnanti, T.L., Wong, R.T.: Network Design and Transportation Planning: Models and Algorithms. Transportation Science 18, 1-55 (1984)

13. Matoušek, R., and Žampachová, E.: Promising GAHC and HC12 algorithms in global optimization tasks. Optimization Methods \& Software 26(3), 405-419 (2011)

14. Pluháček, M., Šenkeř́k, R., and Zelinka, I.: Particle swarm optimization algorithm driven by multichaotic number generator. Soft Computing 18(4), 631-639 (2014)

15. Steenbrink, P.A.: Optimization of Transport Network. John Wiley, New York, USA (1974)

16. Stodola, P., Mazal, J., Podhorec, M., and Litvaj, O.:Using the ant colony optimization algorithm for the capacitated vehicle routing problem. 16th International Conference on Mechatronics - Mechatronika (ME), 503-510 (2014)

17. Šenkeřík, R., Pluháček, M., Davendra, D., Zelinka, I., and Janoštík, J.: New adaptive approach for multi-chaotic differential evolution concept. Hybrid Artificial Intelligent Systems, 234-243, Springer International Publishing (2015)

18. Šoustek, P., Matoušek, R., Dvořák, J., and Bednář, J.: Canadian traveller problem: A solution using ant colony optimization. 19th International Conference on Soft Computing MENDEL 2013, 439-444. Brno, Czech Republic (2013)

19. Šomplák, R., Pavlas, M., Kropác, J., Putna, O., and Procházka, V.: Logistic modelbased tool for policy-making towards sustainable waste management. Clean Technologies and Environmental Policy 16(7), 1275-1286 (2014)

20. Štěpánek, P., Lániková, I., Šimůnek, P., and Girgle, F.: Probability based optimized design of concrete structures. In Life-Cycle and Sustainability of Civil Infrastructure System. London, Taylor \& Francis Group, 2345-2350 (2012)

21. Štětina, J., Klimeš, L., Mauder, T., and Kavička, F.: Final-structure prediction of continuously cast billets. Materiali in tehnologije 46(2), 155-160 (2012)

22. Yo, H., and Solvang, W.D.: A general reverse logistics network design model for product reuse and recycling with environmental considerations. International Journal of Advanced Manufacturing Technology, 1-19 (2016)

23. Zhao, J., Huang, L., Lee, D.-H., and Peng, Q.: Improved approaches to the network design problem in regional hazardous waste management systems. Transportation Research Part E 88, 52-75 (2016) 